\title{
Numerical Study on the Influence of Length-Diameter Ratio on the Performance of Dynamic Pressure Oil-Air Separator
}

\author{
Xiaobin Zhang $\left(\mathbb{D},{ }^{1}\right.$ Xiaofeng Zhang $\mathbb{D}^{1},{ }^{1}$ Delin $\mathrm{Gu}\left(\mathbb{D},{ }^{1}\right.$ Lei Lang $(\mathbb{D}),{ }^{1}$ and Na Gao $\mathbb{( D}^{2}$ \\ ${ }^{1}$ College of Aerospace and Civil Engineering, Harbin Engineering University, Harbin 150001, China \\ ${ }^{2}$ AVIC Aerodynamics Research Institute, Harbin 150001, China \\ Correspondence should be addressed to Delin Gu; gudelin@hrbeu.edu.cn
}

Received 16 November 2020; Revised 9 May 2021; Accepted 22 May 2021; Published 7 June 2021

Academic Editor: Ho SoonMin

Copyright ( 92021 Xiaobin Zhang et al. This is an open access article distributed under the Creative Commons Attribution License, which permits unrestricted use, distribution, and reproduction in any medium, provided the original work is properly cited.

In order to study the separation characteristics of the aeroengine dynamic pressure oil-air separator, this paper uses the coupling method of PBM and CFD two-fluid model to study the influencing factors such as cylinder diameter, cylinder length, and other factors on the separator performance. The flow field structure, velocity, gas volume distribution, separation efficiency, and gas and liquid holdup rate in the separator under different operating conditions are analyzed. Combined with the analysis results of the cylinder diameter and the cylinder length, the influence law of length-diameter ratio on separation efficiency is summarized. The optimum length-to-diameter ratio that maximizes the separation performance of the separator is obtained in this research, which provides a reference for the design and improvement of the separator. The results show that, as the diameter of the cylinder increases, the separation efficiency increases first and then decreases. When $d_{\text {sep }}=16 \mathrm{~mm}$ and $d_{\text {sep }}=18 \mathrm{~mm}$, the separator reaches its maximum efficiency, which is about $93 \%$. With the increase of the cylinder length, the separation efficiency first increases and reaches the maximum when $l_{2}=90 \mathrm{~mm}$ and then decreases slowly. When the separator cylinder is either too long or too short, it will cause the separation performance to decrease. There is an optimal aspect ratio. There is an optimal aspect ratio, and the separation performance of the separator is the best when the aspect ratio is between 5 and 6 .

\section{Introduction}

In a high temperature, high pressure, and high-speed operating environment, the engine requires a high-performance lubrication system to ensure its safe and smooth operation. Due to the overall requirements of the engine's small size and lightweight, the design requirements for the various components of the lubricating oil system have become more strict. After years of hard work, great progress has been made in engine lubrication system research, but it is generally limited to the general theoretical description of the lubrication system or specific failure analysis, and the experimental research is usually limited to the overall performance testing of a certain part in the lubrication system. There is a lack of research on heat exchange, friction, resistance components, and so on. One cannot build their own design models of a component.

During the operation of the engine, clean and lowtemperature lubricating oil is transferred to the rotating parts of the engine, thus reducing friction and taking away the heat generated by friction. Air will enter the lubrication system due to the difference in pressure between the inside and outside. Under the action of high-speed rotating parts, the lubricating oil is mixed with air to become lubricating oil emulsion, which is transported to the oil return system by the oil return pump, which will increase the pipeline resistance, reduce the performance of the oil-fueled radiator, and affect the lubrication conditions of the opposite surface, thereby seriously affecting the safety of the engine. Therefore, an oil and gas separation device is required in the oil return subsystem. Due to the advantages of its simple structure and lightweight structure, the dynamic pressure oil separator has been widely used in small aeroengines [1]. But its flow field is more complicated, involving anisotropic three-dimensional rotating flow, and there are a large number of local secondary flows, such as central return flow, local circulation flow, local short-circuit flow, and other flow 
phenomena. At the same time, due to the existence of the two-phase mixture of oil and gas, the flow field also has a complex change of the two-phase interface. All these factors increase the difficulty of the separator design and optimization process. There are limitations to research on dynamic pressure oil and gas separators in the aviation field. In recent years, the research on dynamic pressure separation of oil and gas has mainly focused on the Gas-Liquid Cylindrical Cyclone (GLCC), discussing the flow characteristics and mechanical behavior of the separator.

In terms of experimental research, Gomez et al. [2] tested the distribution of the subvelocity field in the separator and the changes of the turbulence-related quantities at different positions of the separator. Hreiz $[3,4]$ conducted in-depth experiments on the swirling characteristics of the internal flow field of the tubular gas-liquid separator, discussed the influence of the inlet shape on the flow field and working performance, and proved that the rectangular tapered inlet could effectively improve the performance of the separation. Hsiao [5] discussed the influence of the inlet form on the flow field of the separator and found that the change of the inlet form directly affected the internal flow pattern of the separator, thus affecting the separation efficiency of the separator. Fan et al. [6] used PIV to measure the internal flow field of the separator. By comparing the axial velocity, radial velocity, and separation efficiency, they discussed the influence of different inlet angles on the separator.

With the rapid development of computer technology, numerical simulation methods have been widely used in the research of separators. Miguel A Reyes-Gutiérrez [7, 8] analyzed the single-phase and two-phase flow fields in the separator and discussed the influence of flow and structure parameters on the separation performance. The results showed that the separation efficiency was greatly affected by the behavior of the separator gas core. The installation of an annular membrane device could effectively improve the separation efficiency of GLCC. Saidi et al. $[9,10]$ used the LES model and Lagrangian particle tracking method to study the flow field and separation efficiency of the separator and further discussed the influence of the cone angle on the separation performance and droplet residence time. Meng et al. [11] used the RNG $\kappa-\varepsilon$ model and DPM model to calculate the flow field and oil droplet trajectory of the cyclone separator and studied the velocity distribution, pressure distribution, and separation efficiency of the cyclone separator. Guizani et al. [12] simulated the flow field distribution and pressure drop distribution in the separator through the RSM model and analyzed the influence of the rotor speed on the pressure drop. The results showed that increasing the speed would increase the speed in the countercurrent zone and reduce the separation performance. Yang et al. [13] established the bubble motion trajectory equation from a theoretical perspective and studied the mechanical behavior of the bubble in the separator. In addition, Lu et al. [14] used the DPM model to study the movement of oil droplets in the cyclone separator and calculated the oil droplet size distribution using a semiempirical formula. The results showed that the splashing phenomenon of oil droplets in the separator would cause the decline of the separation performance decline. Guizani et al. [15] simulated the velocity field distribution in the separator by combining the RSM model and the DPM model, and the simulation results better reflected the flow field distribution law. Van et al. [16] used RSM to discuss the effect of inlet angle on single-phase flow field in the separator and presented the differences in the distribution of the flow field under different situations and the improved design of the separator structure. Wang et al. [17] also used RSM to simulate the cyclone field in the separator and obtained the pressure drop and velocity distribution rules of the flow field in the separator, and the simulation results were basically consistent with the experimental results. On this basis, Zhu et al. [18] studied the outlet forms at the bottom of the separator by using RSM, discussed the influence of different outlet forms on the flow field distribution, and believed that the single tangential outlet is more conducive to improving the separation efficiency. Ghasemi et al. [19] conducted a numerical simulation of the separator using the Eulerian model, discussed the influence of structural factors such as inlet width, inlet angle, inlet height, cylinder diameter, and outlet pipe diameter on the separation efficiency, and proposed a structural optimization scheme. Li et al. [20] used the PBM model to conduct numerical simulation of the flow field in the agitator and compared the simulation results of five different droplet breaking models on the droplet breaking process. Siadaty and Huang et al. [21, 22] used the Eulerian-Lagrangian method to conduct a comprehensive study on the effects of airflow and structure on the performance of cyclones. The analysis results showed that the higher the temperature, the weaker the swirling flow, and the flow pressure drop and particle separation efficiency were significantly reduced. At the same time, increasing the air gap width and reducing the exhaust angle of the guide vane could improve separation efficiency. Li et al. [23] took the axial cyclone separator with an inner diameter of $150 \mathrm{~mm}$ as the research object and studied the influence of material humidity on its inlet particle size distribution, overall efficiency, classification efficiency, and cutting particle size. It was found that when the inlet velocity and the particle humidity were constant, due to the aggregation of the particles, as the particle concentration increases, the efficiency of both the collection and classification was significantly improved. Yue et al. [24] used experiments and numerical simulations to explain the flow state and flow behavior of the upper spinning liquid film (USLF) in the gasliquid cylindrical cyclone. According to the experiment, the four flow patterns were determined: swirling flow, stirring flow, annular flow, and ribbon flow. Their flow pattern diagrams were also established. Yang et al. [25] also combined experiments and numerical analysis in the study of the separation characteristics of the gas-liquid cylindrical cyclone. The results showed that a separator with a strong swirling flow might not be able to achieve a better separation effect. Finally, based on the force of the droplet and the swirling dynamics, a droplet migration model was established and accurately predicted the separation performance of the droplet. Based on the aeroengine lubricating oil system test bench, Zhang [26] used a dimensional analysis 
method to establish a mathematical model for predicting the separation efficiency and resistance of a dynamic pressure oil-air separator suitable for engineering. The analysis of the multivariate nonlinear fitting error and the experimental data showed that the established separation efficiency and resistance model could accurately predict the separation and resistance performance of the dynamic pressure oil-air separator within a certain range.

Generally speaking, most of the previous researches were mainly focused on hydrocyclone separators in industrial fields such as petrochemicals and bioengineering. However, due to factors such as small inlet flow and limited installation space, the structural dimensions of aeroengine hydrocyclone separators are quite different. This results in differences in the flow field of the separator, and the influence of the structure on the flow field will also change. Therefore, the research results on hydrocyclones are difficult to be applied directly to the aerospace field. In speaking of aeroengine special separator, although numerical simulation can clearly express the flow situation inside the separator, the physical model proposed by most current researches is still not perfect. Therefore, in order to study the performance of the aeroengine dynamic pressure oil-air separator, this paper adopts the method of coupling the PBM and CFD two-fluid model. First, the accuracy of the method is verified by comparing with the experimental results, and then the cylinder diameter, cylinder length, and other factors are studied. The impact on the performance of the separator, the flow field structure, velocity, gas volume distribution, separation efficiency, and gas-liquid content of the separator under different working conditions is analyzed. Finally, the two influencing factors are combined and obtained within the scope of this paper. An optimal length-to-diameter ratio that optimizes the separation performance of the separator provides a reference for the design and improvement of the separator.

\section{Numerical Model}

2.1. Turbulence Model Selection and Verification. The dynamic pressure oil and gas separator has a two-phase strong turbulence field inside, so the research should determine the turbulence model first. Then, the two-phase flow model can be determined before carrying out any relevant research work. However, since the author did not have any separate experimental data of the turbulence, the scholars in literature [27] carried out experimental and numerical research on the gas-liquid cyclone separator and obtained the experimental data. Thus, after the experimental data in literature [27] were selected to compare and analyze the three turbulence models, the turbulence model was selected.

Based on the physical modeling of the cyclone separator provided in literature [27], this paper uses different turbulence models to calculate its internal single-phase flow field and compares the calculated cross-sectional velocity with the experimental data in the literature. For comparison, the most suitable turbulence model is selected. The physical model in the literature is shown in Figure 1. In addition, due to the length limit, the three turbulence models are not described in this paper. For the details of the three models, please refer to literature [28].

The dimensionless axial velocity distribution on different sections is shown in Figure 2. $U_{\mathrm{av}}$ represents the axial velocity, $r$ is the position from any position to the center, and $R$ is the radius of the separator. It can be seen from the figure that the calculation results of different turbulence models are different. In Section 1, the area on the left of the coordinate axis, the Realizable $k-\varepsilon$ calculated values of the three turbulence models are in good agreement with the experimental values; the area on the right of the coordinate axis is calculated by the Realizable model. The calculated axial velocity value is closer to the experimental value, with the smallest error. Similarly, in Section 2 , the Realizable $k-\varepsilon$ calculation result of the $\mathrm{Re}$ alizable $k-\varepsilon$ model is also the closest to the experimental value. Figure 3 shows the dimensionless tangential velocity distribution in each section, and $u_{\mathrm{t}}$ represents the tangential velocity. In Section 1, the tangential velocity values Realizable $k-\varepsilon$ calculated by the three turbulence models all present a center-symmetric distribution, and the tangential velocity values Realizable $k-\varepsilon$ calculated by the Realizable model are closer to the experimental values Realizable $k-\varepsilon$ with the smallest error. In Section 2, it can also be seen that the calculation results of the Realizable model are closer to the experimental values, followed by the RNG $k-\varepsilon$ model, and the standard turbulence model has the largest error. In summary, the Realizable $k-\varepsilon$ model has a better predictive effect on the gas-liquid cyclone flow field in the separator and can more accurately obtain the axial and tangential velocity distributions on different sections. The results are consistent with those obtained in literature [27]. The results are the same, which provides a guarantee for the study of flow field distribution and its separation mechanism.

2.2. Two-Phase Flow Model. In order to calculate the twophase flow state in the separator, the two-phase flow model shall be determined after selecting the appropriate turbulence model in the previous section. In this paper, the coupling method of CFD and PBM dual-fluid model is adopted to study the variation of the separator's performance under different working conditions. Therefore, this section mainly verified the accuracy of the two-phase flow model by combining the experimental data. The Euler-Euler two-fluid model can consider comprehensively the turbulent transport of particle phase, and the particle phase (gas phase) and continuous phase (liquid phase) can be treated reasonably. Compared with the Euler-Lagrange method, the Euler-Euler model assumes that the bubble discrete phase is quasifluid, which has a lower computation capacity. PBM model takes into account the influence of bubble polymerization and crushing behavior on the bubble size distribution and is able to predict the flow field distribution of gas-liquid separation well, which has been widely used in the study of gas-liquid separation. Therefore, this paper combines CFD and PBM two-phase flow model to conduct numerical simulation of the internal flow field of the dynamic pressure oil and gas separator to ensure the accuracy of the calculation results. 

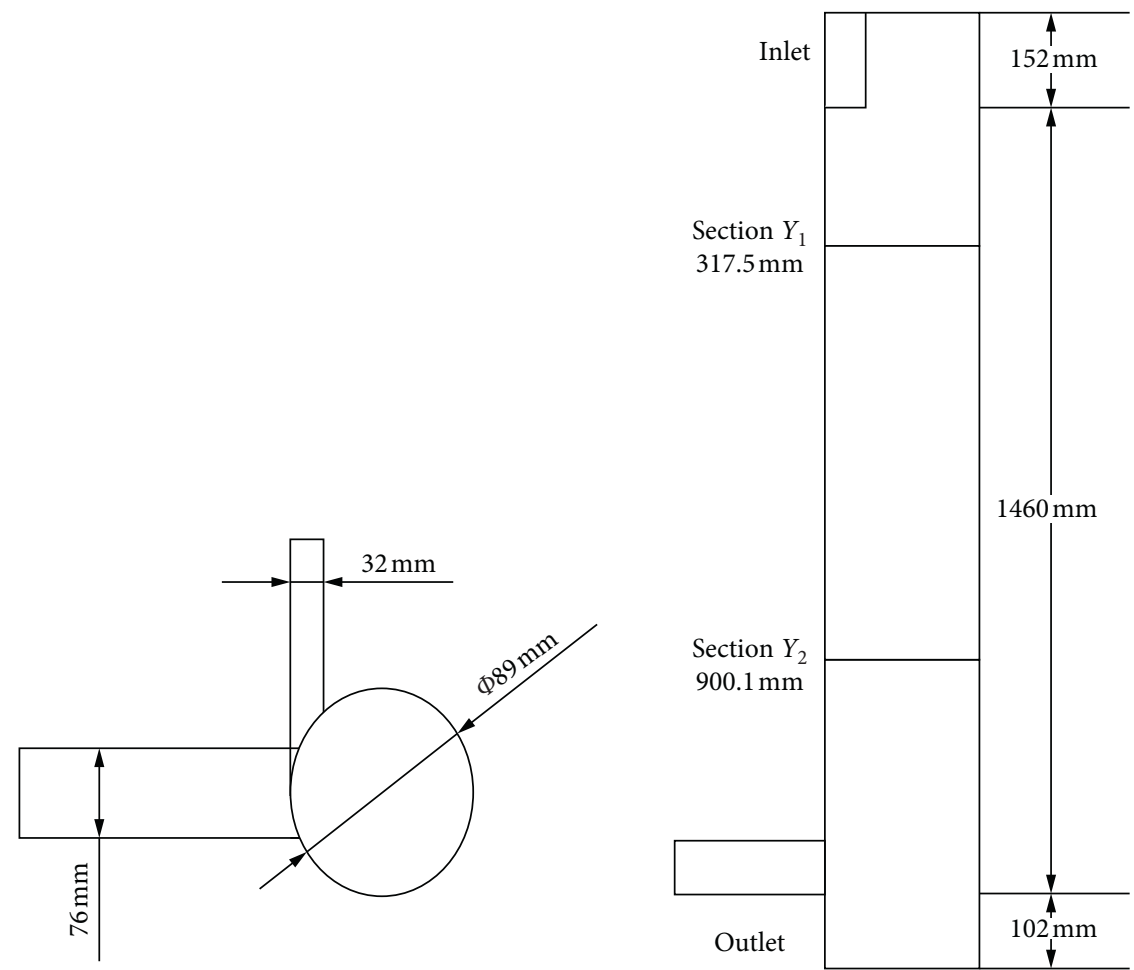

Figure 1: Cyclone separator model.

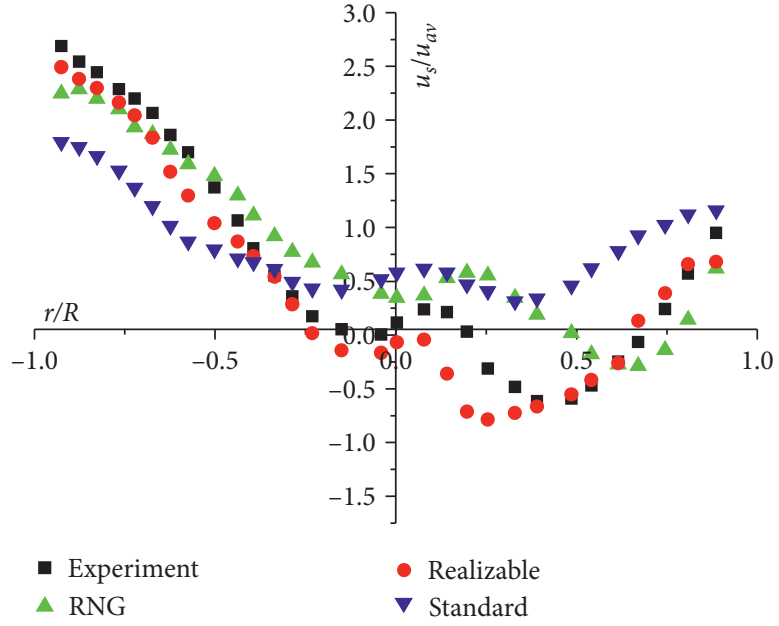

(a)

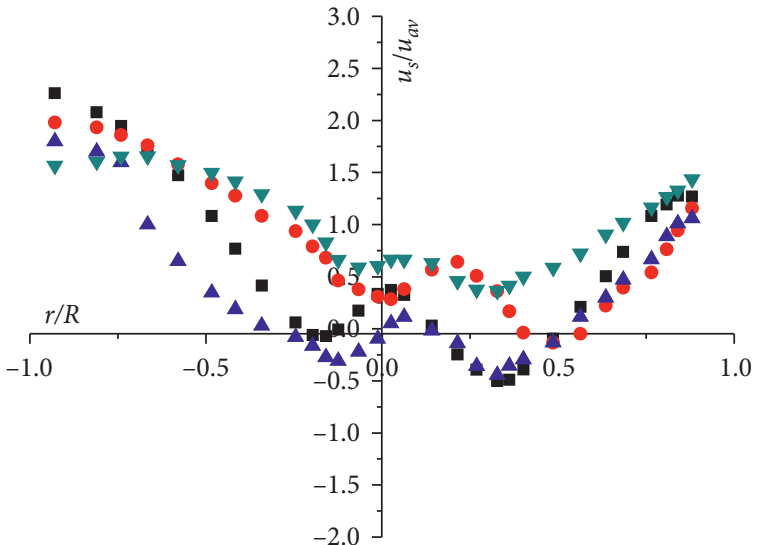

- Experiment

- RNG
A Realizable
$\nabla$ Standard

(b)

Figure 2: Axial velocity of each section. (a) Section 1. (b) Section 2.

2.2.1. Two-Fluid Equation. In this paper, the Euler-Euler method is used to solve and analyze its swirling flow field, and both phases are used as continuous fluids to solve its motion equations. In the calculation process, the bubble is considered a uniform sphere, and there is mutual penetration between the oil and gas phases, filling the entire separator space and neglecting the mass and energy transfer between each other.

Continuity equation is as follows:

$$
\begin{aligned}
\nabla \cdot\left(\alpha_{l} \rho_{l} \mathbf{u}_{l}\right) & =0, \\
\nabla \cdot\left(\alpha_{g} \rho_{g} \mathbf{u}_{g}\right) & =0, \\
\alpha_{l}+\alpha_{g} & =1 .
\end{aligned}
$$

In the formula, $\alpha_{i}$ and $\alpha_{g}$ represent the liquid and gas volume fractions; $\rho_{i}$ and $\rho_{g}$ represent the liquid and gas density, $\mathrm{kg} / \mathrm{m}^{3} ; \mathbf{u}_{i}$ and $\mathbf{u}_{g}$ represent the liquid and gas velocity, $\mathrm{m} / \mathrm{s}$. 


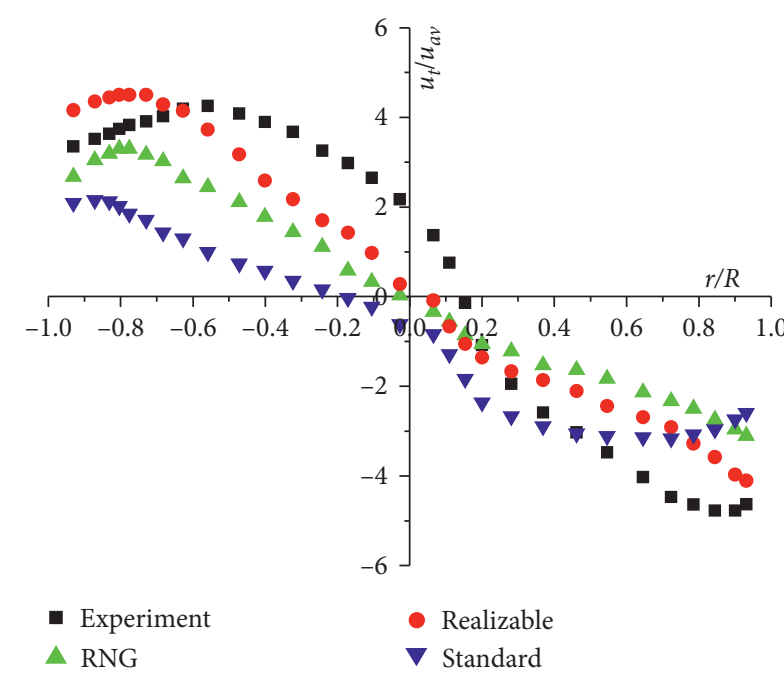

(a)

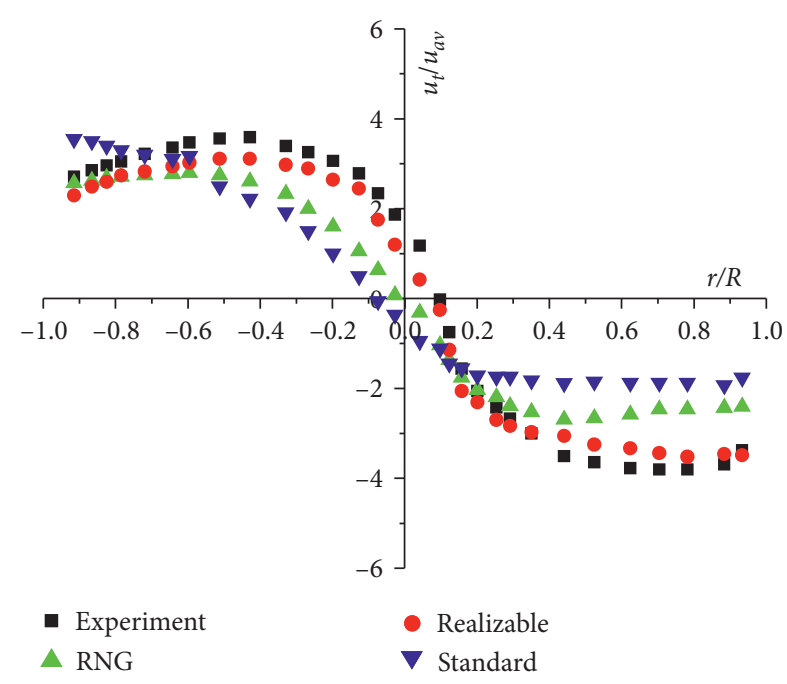

(b)

Figure 3: Tangential velocity of each section. (a) Section 1. (b) Section 2.

Momentum equation is as follows:

$$
\nabla \cdot(\rho \alpha \mathbf{u u})_{j}=-\alpha_{j} \nabla \mathbf{P}+\nabla \cdot\left[\alpha \mu_{\mathrm{eff}}\left(\nabla \mathbf{u}+\nabla \mathbf{u}^{T}\right)\right]_{j}+(\rho \alpha)_{j} g+\mathbf{F}_{a, o} .
$$

In the formula, $j$ represents $j$-th phase fluid type; $u$ represents the speed of each phase, $\mathrm{m} / \mathrm{s} ; \mathbf{P}$ represents the pressure, $\mathrm{Pa} ; \mu_{\text {eff }}$ represents the effective viscosity coefficient of the $j$-th phase, Pa.s; $\mathbf{F}_{a, o}$ represents the interaction force between the two phases, $\mathrm{N}$.

2.2.2. Group Balance Equation. In order to calculate the twophase flow state in the separator, this paper selects the group balance model (PBM model) for calculation. The PBM model considers the influence of bubble aggregation and breaking behavior on bubble size distribution and can better predict the distribution of gas-liquid separation flow field, and it has been widely used in the study of gas-liquid separation problems. Its specific expression form is shown in formula (3):

$$
\frac{\partial}{\partial t}[n(V, t)]+\nabla\left(u_{i} n(V, t)\right)=B_{i}^{C}+B_{i}^{B}-D_{i}^{C}-D_{i}^{B},
$$

where $i$ represents the $i$-th group of bubbles, $n(V, t)$ is the distribution function of the volume of $\mathrm{V}$ bubbles in unit time and unit space; $u_{i}$ is the movement speed of the $i$-th group of bubbles; $B_{i}^{C}$ and $B_{i}^{B}$ represent the amount of bubble generation caused by bubble aggregation and fragmentation; $D_{i}^{C}$ and $D_{i}^{B}$ represent the amount of bubble extinction caused by bubble aggregation and breaking.

(1) The amount of formation caused by bubble breakage is as follows:

$$
B_{i}^{B}=\int_{\Omega_{v}} p g\left(V^{\prime}\right) \beta\left(V \mid V^{\prime}\right) n\left(V^{\prime}, t\right) \mathrm{d} V^{\prime} .
$$

In the formula, $p$ represents the number of subbubbles generated by bubble breaking; $g\left(V^{\prime}\right)$ represents the bubble breaking frequency with volume $V^{\prime}, \mathrm{m}^{3} / \mathrm{s} ; \beta\left(V \mid V^{\prime}\right)$ represents the probability density function of subbubbles formed by the breaking of bubbles with volume in $V^{\prime} \sim V ; n\left(V^{\prime}, t\right)$ represents the distribution function of bubbles with volume $V^{\prime}$.

(2) The amount of extinction caused by bubble break is as follows:

$$
D_{i}^{B}=g(V) n(V, t) .
$$

In the formula, $g(V)$ represents the breaking frequency of bubble with volume $V, \mathrm{~m}^{3} / \mathrm{s}$.

(3) The amount of formation caused by bubble aggregation is as follows:

$B_{i}^{C}=\frac{1}{2} \int_{0}^{v} a\left(V-V^{\prime}, V^{\prime}\right) n\left(V-V^{\prime}, t\right) n\left(V^{\prime}, t\right) d V^{\prime}$.

In the formula, $a\left(V-V^{\prime}, V^{\prime}\right)$ represents the frequency at which bubbles of volume $V-V^{\prime}$ and $V^{\prime}$ the coalesce, $\mathrm{m}^{3} / \mathrm{s} ; n\left(V-V^{\prime}, t\right)$ represents the distribution function of bubbles with volume $V-V^{\prime}$.

(4) The amount of extinction caused by bubble aggregation is as follows:

$$
D_{i}^{c}=\int_{0}^{\infty} a\left(V, V^{\prime}\right) n(V, t) n\left(V^{\prime}, t\right) d V^{\prime}
$$

In the formula, $a\left(V, V^{\prime}\right)$ represents the volume $V$ at which bubbles of volume $V$ and $V^{\prime}$ coalesce, $\mathrm{m}^{3} / \mathrm{s}$. In addition, the bubble breaking and aggregation model proposed in literature $[29,30]$ are used to solve equations (4) (7), which will not be repeated here. 
2.3. Meshing. According to literature [31], the corresponding physical model of the dynamic pressure oil-gas separator is established, as shown in Figure 4. Its specific structure parameter definition and size are shown in Table 1.

The physical model in Figure 4 is meshed, and the entrance and exit faces of the separator are meshed and refined. The results are shown in Figure 5. In order to clearly show the flow field inside the separator, cross-section $Y_{3}$ is taken at $50 \mathrm{~mm}$ in the axial direction.

In order to verify the independence of the grid, this paper uses seven grid numbers to calculate the flow field of the separator and obtains the law of the influence of different grid numbers on the separation efficiency of the separator. The results are shown in Figure 6. It can be seen from Figure 6 that when the number of grids is greater than 550,000 , the separation efficiency and pressure distribution calculated at each flow rate basically remain unchanged. The specific calculation form of separator efficiency is shown in equations (10) (12). Therefore, in order to save resources and shorten the calculation time as much as possible, the grids used for all the subsequent calculations are about 550,000 .

2.4. The Boundary Conditions and Solution. The end of the separator inlet pipe is used as the velocity inlet, and the gas and liquid outlets are pressure outlets and are set as atmospheric, as shown in Figure 5. The density and viscosity of the lubricating oil used in the simulation are $972.2 \mathrm{~kg} / \mathrm{m}^{3}$ and $0.036134 \mathrm{~Pa} \cdot \mathrm{s}$, respectively. The inlet velocity is $1.27 \mathrm{~m} / \mathrm{s}$, and the corresponding flow rate is $6 \mathrm{~L} / \mathrm{min}$. The air physical property parameter is the constant physical property under constant temperature, and the oil-air ratio is 1 . At this point, the Reynolds number at the inlet is 8357. Turbulence intensity $I$, turbulence kinetic energy $k$, and turbulence dissipation were also set for calculation, which are calculated using equations (8) and (9). They were 0.052, 0.007, and 0.03, respectively.

$$
\left.\begin{array}{l}
I=0.16 \cdot R_{e}^{-0.125}, \\
R_{e}=\frac{D_{H} u \rho}{\mu},
\end{array}\right\}
$$

where $D_{H}$ represents the inlet hydraulic diameter, $\mathrm{m} ; u$ represents the inlet velocity, $\mathrm{m} / \mathrm{s} ; \rho$ represents the density, $\mathrm{kg} / \mathrm{m}^{3}$; and $\mu$ represents the dynamic viscosity, Pa.s.

$$
\left.\begin{array}{l}
k=\frac{3}{2}(u I)^{2}, \\
\varepsilon=C_{\mu}^{3 / 4} \frac{k^{3 / 2}}{l},
\end{array}\right\}
$$

where $u$ represents the inlet velocity, $\mathrm{m} / \mathrm{s} ; l$ represents the turbulence characteristic size, $\mathrm{m}$; and $C_{\mu}$ represents the empirical constant, 0.09 .

In the calculation, the wall surface adopts the nonslip boundary condition. Because the near-wall area is affected by the viscous effect, the fluid velocity is low, the turbulent diffusion is weak, and the turbulent transport equation is not strictly valid. Therefore, the standard wall function is used to solve the flow field near the wall. At the same time, bubbles in the range of $0.001 \mathrm{~mm}$ to $1 \mathrm{~mm}$ are divided into 10 groups by their sizes to determine the calculation conditions of the numerical simulation [31].

2.5. Model Validation. In order to evaluate the accuracy of the calculation results obtained by solving the PBM model in the unsteady state, the calculated separation efficiency is compared with the experimental values in literature [31]. The numerical simulation uses the same working conditions and structural parameters as the experiment, and the comparison result is shown in Figure 7. It can be seen that, for different separators, the variation trend of the separation efficiency measured by the numerical calculation and the experiment at each flow rate is consistent, and the average relative error under each working condition is less than $15 \%$. Therefore, it is believed that the PBM model can be used for the calculation of the gas-liquid cyclone separation problem.

\section{Results and Discussion}

In the process of studying the influence of the length-diameter ratio on separation performance, it is particularly important to evaluate the performance of the separator. For the gas-liquid cyclone separator, its performance is often measured by two parameters: gas holdup rate and liquid holdup rate. The liquid holdup rate represents oil entrapment at the gas outlet. The gas holdup rate represents gas entrainment at the oil outlet [4]. Therefore, a good separator should not only have a reasonable flow field structure but also have lower gas and liquid holdup rate. The liquid holdup rate is determined by equation (10), and the gas holdup rate is shown in equation (11).

$$
k_{1}=\frac{q_{l 1}}{q_{l}}
$$

where $q_{l 1}$ represents the volume flow rate of lubricating oil at the air outlet, L/min; $q_{l}$ represents the volume flow rate of lubricating oil at the inlet, $\mathrm{L} / \mathrm{min}$.

$$
k_{2}=\frac{q_{g 1}}{q_{g}},
$$

where $q_{g 1}$ represents the volume flow rate of air at oil outlet, $\mathrm{L} / \mathrm{min} ; q_{g}$ represents the volume flow rate of air at the inlet of separator, L/min. Although $K_{2}$ can reflect the separation performance of the separator, it is not intuitive, so the separation efficiency of the separator is defined, as shown in equation (12).

$$
\eta=1-k_{2}
$$

where, $\eta$ represents the separation efficiency of the separator.

3.1. Flow Field Analysis of Separator. In order to analyze the flow field structure of the separator in detail, the plane is intercepted along its central position, and its internal flow is 

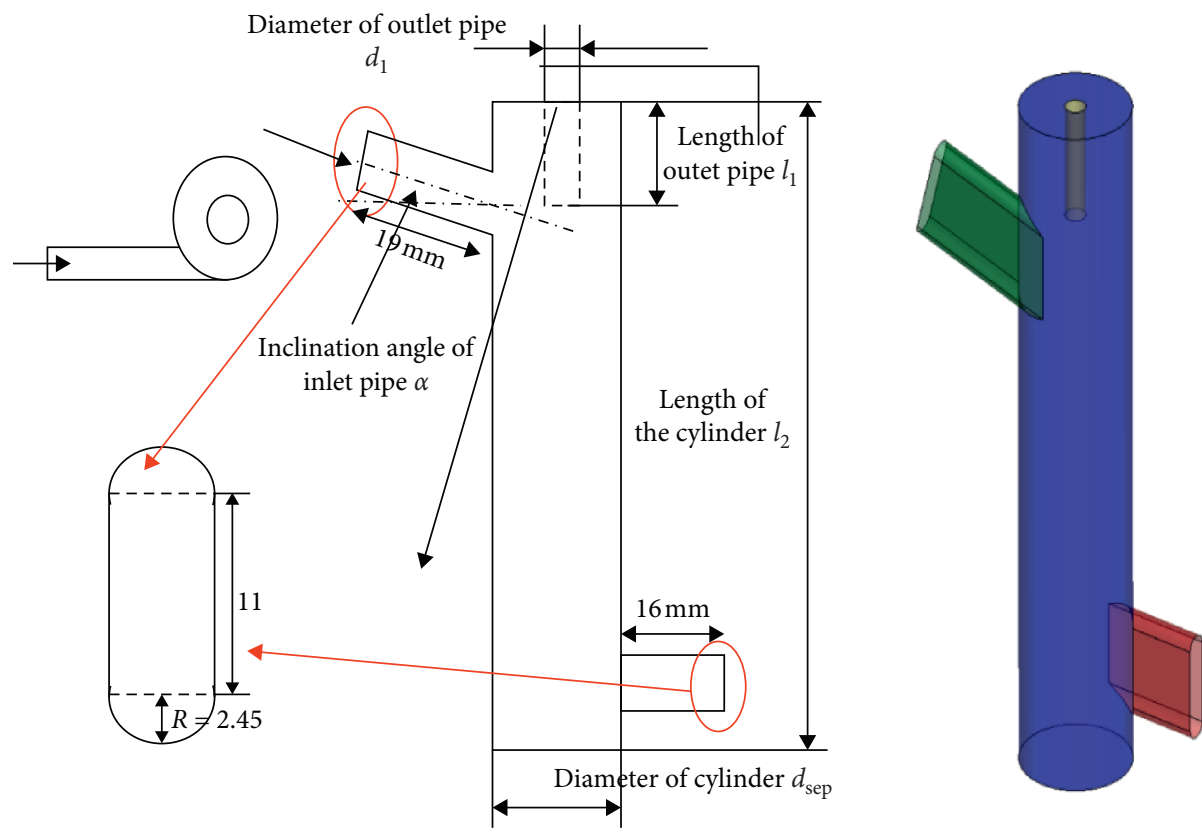

FIgURE 4: Structure diagram of separator.

TABLE 1: Structure parameters of separator.

Name

Length of air outlet $l_{1}(\mathrm{~mm})$

Length of cylinder $l_{2}(\mathrm{~mm})$

Outlet pipe diameter $d_{1}(\mathrm{~mm})$

Cylinder diameter $d_{\text {sep }}(\mathrm{mm})$

Angle of entrance $\alpha\left({ }^{\circ}\right)$

Inlet length $(\mathrm{mm})$

Outlet length $(\mathrm{mm})$

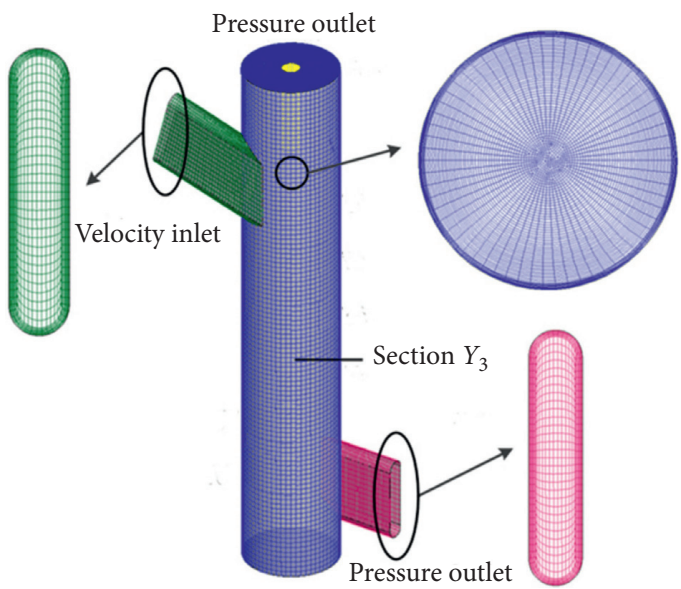

FIgURE 5: Mesh division.

shown in Figure 8. As can be seen from Figure 8, the flow field structure in the separator can be divided into two regions: the downward outer cyclone region near the wall and the upward inner cyclone region in the center (also known as backflow). The radial size of the inner cyclone indicates the separation location of bubbles during operation, which will directly affect the separation performance of the separator. At the same time, it can be seen that the central backflow area has a slight left-right oscillation relative to the axis. Through local amplification, it can be seen that there are many localized, secondary flow phenomena in a small-scaled dynamic pressure type oil and gas separator.

A is the circulation flow at the top of the separator. The two-phase fluid spreads along the wall at the entrance of separator causing part of the fluid to flow directly upwards. It reaches the top of the separator and bounces back. It flows down along the center of the separator, thus forming the circulation flow. B is the short-circuit flow near the gas outlet. Most of the fluid at the inlet moves along the tangential motion towards the bottom of the separator at the same time. The separated lubricating oil travels down the wall and the gas converges in the central region, forming a gas core column. Under the pressure, the gas gets close to the air outlet, which moves in the opposite direction to the lubricating oil in the axial, so the gas core column rolls part of the fluid from the air inlet to the outlet, thus forming a short-circuit flow near the air outlet. (C) is a circular flow at the bottom of the separator, which forms a dual vortex structure with (D). This area increases with the increase of the flow, which is because of the tangential gas outlet structure used in the oil-air separator studied in this paper The downward-flowing fluid hits the wall at the bottom of the cylinder and rebounds upward, forming a local circulation flow. (C) and (D) rotate in opposite directions. On one hand, the gas near the wall of the cylinder is brought into the air core column to enhance the separation effect. On the other hand, some of the gas in the air core column is rolled to 


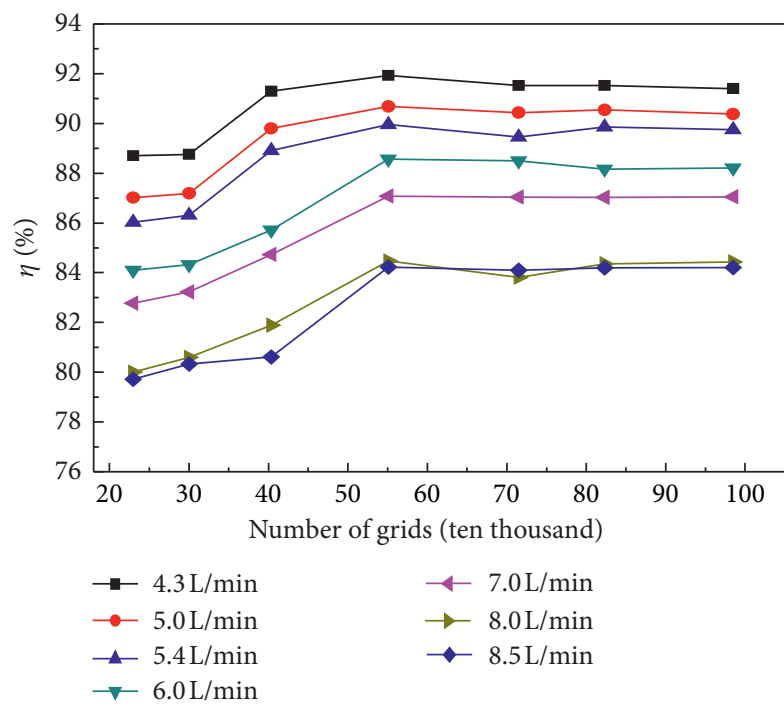

(a)

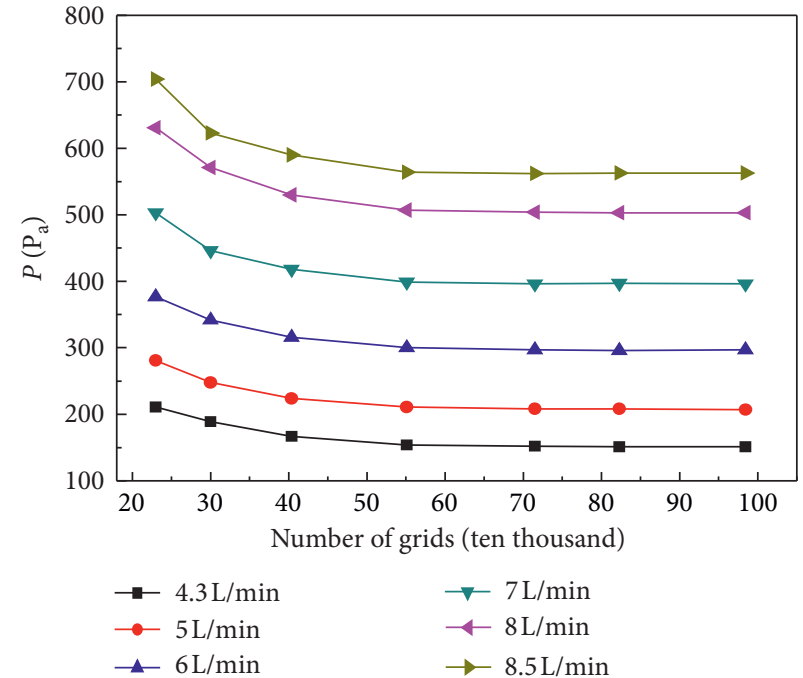

(b)

Figure 6: The effect of the number of grids. (a) Separation efficiency. (b) Pressure distribution.

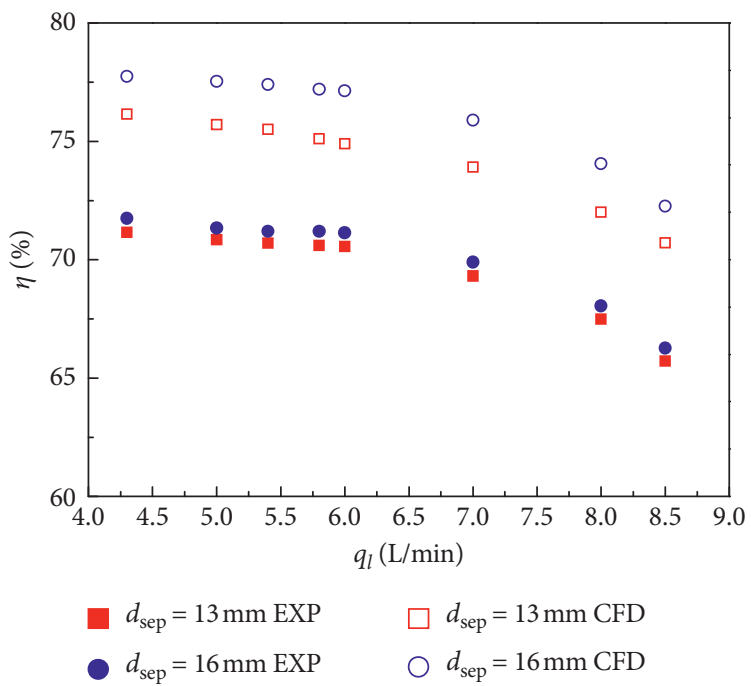

FIgURE 7: Comparison of calculated value of separator with experimental data.

the bottom of the cylinder to reduce the separation performance.

3.2. The Influence of Cylinder Diameter on Separator Performance. Figure 9 shows the structure distribution of flow field structure in the separator under different cylinder diameters. It can be seen from the figure that the diameter of the cylinder directly affects the structure of the flow field. When $d_{\text {sep }}=10 \mathrm{~mm}$, there is a top circulation flow, an outlet pipe short-circuit flow, and a bottom circulation flow inside the separator.

The bottom circulation flow is a single vortex structure, as shown in (A). With the increase of the diameter of the cylinder, the vortex in the central region of the cylinder gradually expands along the axial, and the impact expands (see the comparison of B, C, and D). The bottom circulating flow is split from a single vortex structure into a double vortex structure (see E and F). The separation effect is enhanced. The latter double vortex structure is extruded by the vortex in the central region and recombined into a single vortex structure (see $\mathrm{G}$ and $\mathrm{H}$ ), and the range is gradually reduced.

In addition, as the diameter of the cylinder increases, the top circulating flow is also impacted, which is shifted downward from the top end of the separator (see I), and finally splits into double vertex (see L). Under this influence, a large amount of gas will accumulate at the top of the separator, resulting in the deterioration of separation performance. In summary, from the flow field structure alone, 


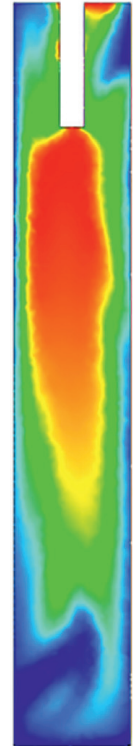

(a)

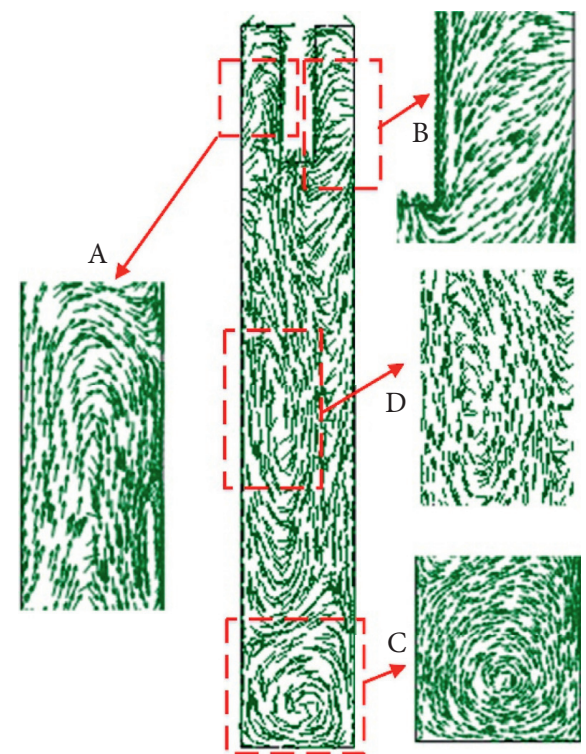

(b)

FIgUre 8: Flow field analysis. (a) Gas volume distribution. (b) Velocity vector.

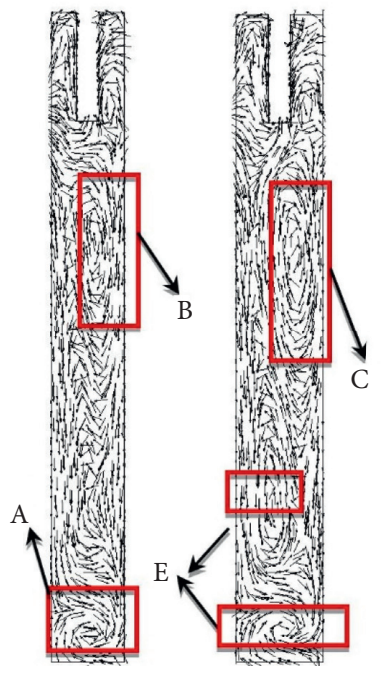

(a)

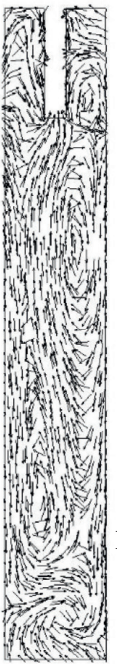

(c)

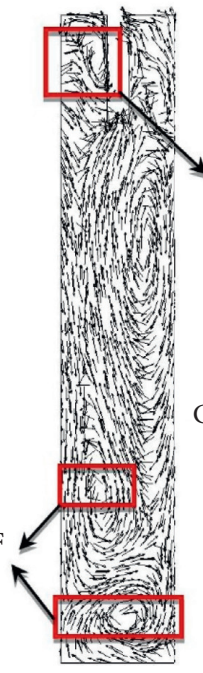

(d)

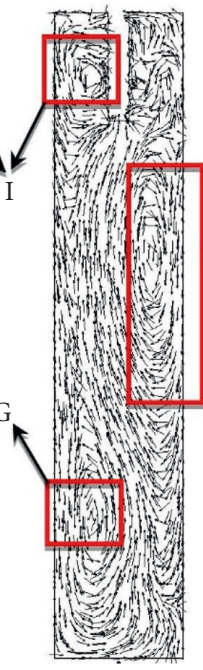

(e)

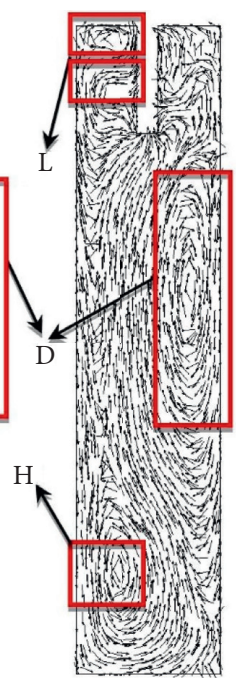

(f)

Figure 9: Separator flow field distribution at different cylinder diameters. (a) $d_{\text {sep }}=10 \mathrm{~mm}$. (b) $d_{\text {sep }}=12 \mathrm{~mm}$. (c) $d_{\text {sep }}=14 \mathrm{~mm}$. (d) $d_{\text {sep }}=16 \mathrm{~mm}$. (e) $d_{\text {sep }}=18 \mathrm{~mm}$. (f) $d_{\text {sep }}=20 \mathrm{~mm}$.

when $d_{\text {sep }} \in[14-16] \mathrm{mm}$, it is more conducive to gas-liquid separation.

Figure 10 shows the axial velocity distribution of the $Y_{3}$ section of the separator under different cylinder diameters. It can be seen from Figure 10(a) that although the diameter of the cylinder is different, the axial velocity in the central area is the largest and gradually decreases with the radial outward; in the area near the wall, the axial velocity gradually increases first and then decreases with the increase in the radial position. Comparing Figures 10 (a) and 10(b), it can be seen that the radial range of the air core column gradually expands due to the influence of the cylinder diameter. In addition, the separated lubricating oil is in the near-wall area and flows downward. It can be clearly seen from the figure that as the cylinder diameter decreases, the lubricating oil in the near-wall area flows down faster, but the medium in the center area is in the case of air, the law is different. When the cylinder diameter increases from $10 \mathrm{~mm}$ to $15 \mathrm{~mm}$, the gas axial velocity becomes larger. If the cylinder diameter continues to increase, the gas axial velocity gradually decreases, indicating that, under the condition that other parameters remain unchanged, there is a certain cylinder 


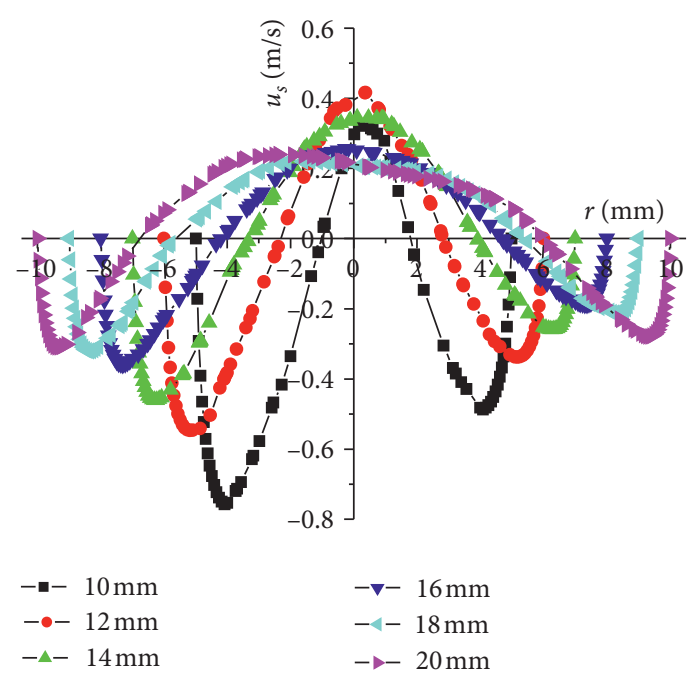

(a)

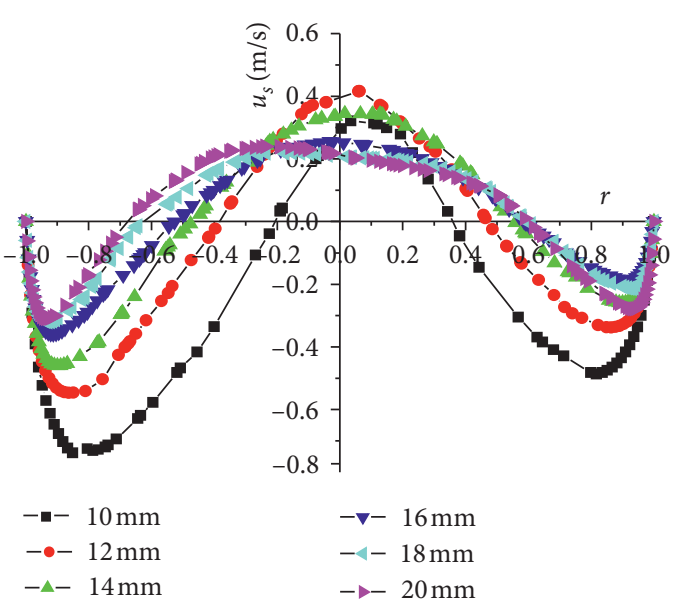

(b)

Figure 10: Axial velocity distribution of $Y_{3}$ section at different cylinder diameters. (a) Absolute radial quantity. (b) Radial dimensionless.

diameter to optimize the separation performance of the separator.

Figure 11 shows the tangential velocity distribution of the $Y_{3}$ section of the separator under different cylinder diameters. It can be seen from the figure that the tangential velocity under each cylinder diameter is distributed in an inverted " $\mathrm{S}$ " shape, with opposite directions on both sides of the center. Due to the influence of the wall boundary layer, it first increases and then decreases in the radial direction. At the maximum value, the tangential velocity at the wall is zero. Enlarging the right side of Figure 11 and combining with Table 2 , it can be clearly seen that the maximum value of the tangential velocity increases first and then decreases with the increase of the cylinder diameter. When $d_{\text {sep }}=16 \mathrm{~mm}$, it is the peak value; the greater the tangential velocity, the better the separation performance. Therefore, from this point of view, $d_{\text {sep }}=16 \mathrm{~mm}$ is more favorable for gas-liquid separation.

In order to more intuitively describe the influence of the diameter of the cylinder on the performance of the separator, a cloud diagram of the gas volume distribution in the separator is drawn, as shown in Figure 12. It can be seen from the figure that the diameter of the cylinder has a great influence on the gas distribution in the separator. An obvious " $\mathrm{S}$ " shape gas core column is formed inside, and only a small amount of gas accumulates at the top, but the bottom circulating flow is a single vortex structure. The influence of the vortex brings a large amount of gas at the end of the gas core column into it, causing the gas volume fraction at the outlet of the separator to be too high, so the separation performance of the separator decreases.

As the diameter of the cylinder increases, the phenomenon of gas accumulation at the top of the separator becomes more serious. When $d_{\text {sep }}=14 \mathrm{~mm}$ and $d_{\text {sep }}=16 \mathrm{~mm}$, a lubricating area (see A) begins to appear under the gas accumulation area on the top cylinder wall.
The gas is prevented from moving down the cylinder wall, the oil content near the outlet pipe adjacent to this area is very low (see B), and the gas can smoothly flow down in the axial direction and be discharged from the outlet pipe to enhance the separation effect. Therefore, combined with the analysis of the flow field structure, it can be considered that the built-in gas outlet pipe can effectively discharge the gas at the top of the separator, thereby enhancing the separation effect of the separator. In addition, as the diameter of the cylinder increases, the air core column gradually evolves from the "S" type to the "M" type, and when $d_{\text {sep }}=14 \mathrm{~mm}$ and $d_{\text {sep }}=16 \mathrm{~mm}$, the shape of the air core column is just better than that of the " $\mathrm{S}$ " and " $\mathrm{M}$ " type. In the transition stage of the type, the bottom circulation flow is a double vortex structure, and the gas content of the oil outlet is lower. From the value in the gas volume distribution cloud chart, $d_{\text {sep }}=16 \mathrm{~mm}$ is more conducive to gas separation. From the above analysis, it can be seen that when the diameter of the separator cylinder changes from $10 \mathrm{~mm}$ to $20 \mathrm{~mm}$, the gas concentration near the oil outlet of the separator increases first and then becomes smaller. In combination with the above, it can be seen that the best aspect ratio is present under the condition determined by the operating condition parameters, which makes the separator the best separation performance.

Figure 13 shows the separation efficiency and distribution of liquid holdup rate distribution of the separator under different cylinder diameters. As the diameter of the cylinder increases, the separation efficiency increases. When $d_{\text {sep }}=16 \mathrm{~mm}$ and $d_{\text {sep }}=18 \mathrm{~mm}$, the separation efficiency of the separator is equivalent, and its value is the largest, about 93\%; at the same time, there is always a liquid entrainment phenomenon inside the separator under different cylinder diameters; the liquid holdup rate is at the lowest when the separation efficiency is maximized, indicating that there is an optimal aspect ratio to make the separator the best 


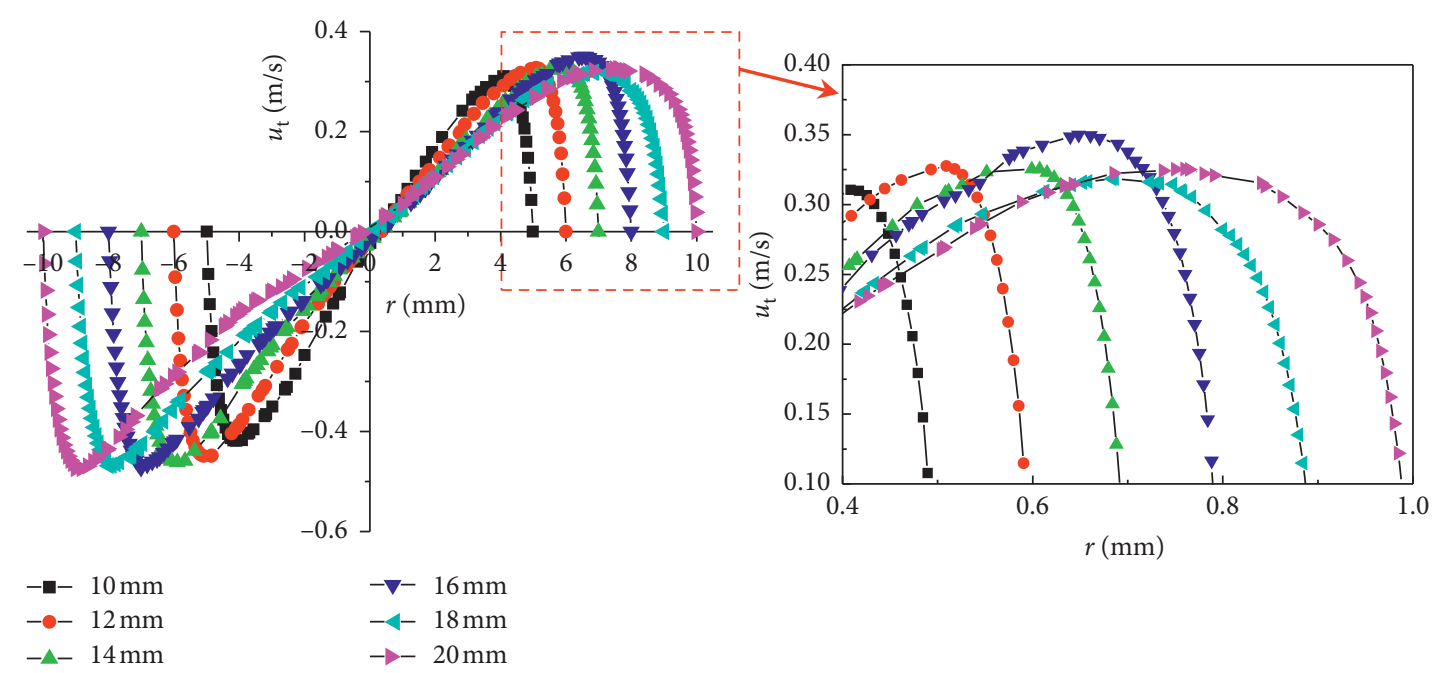

(a)

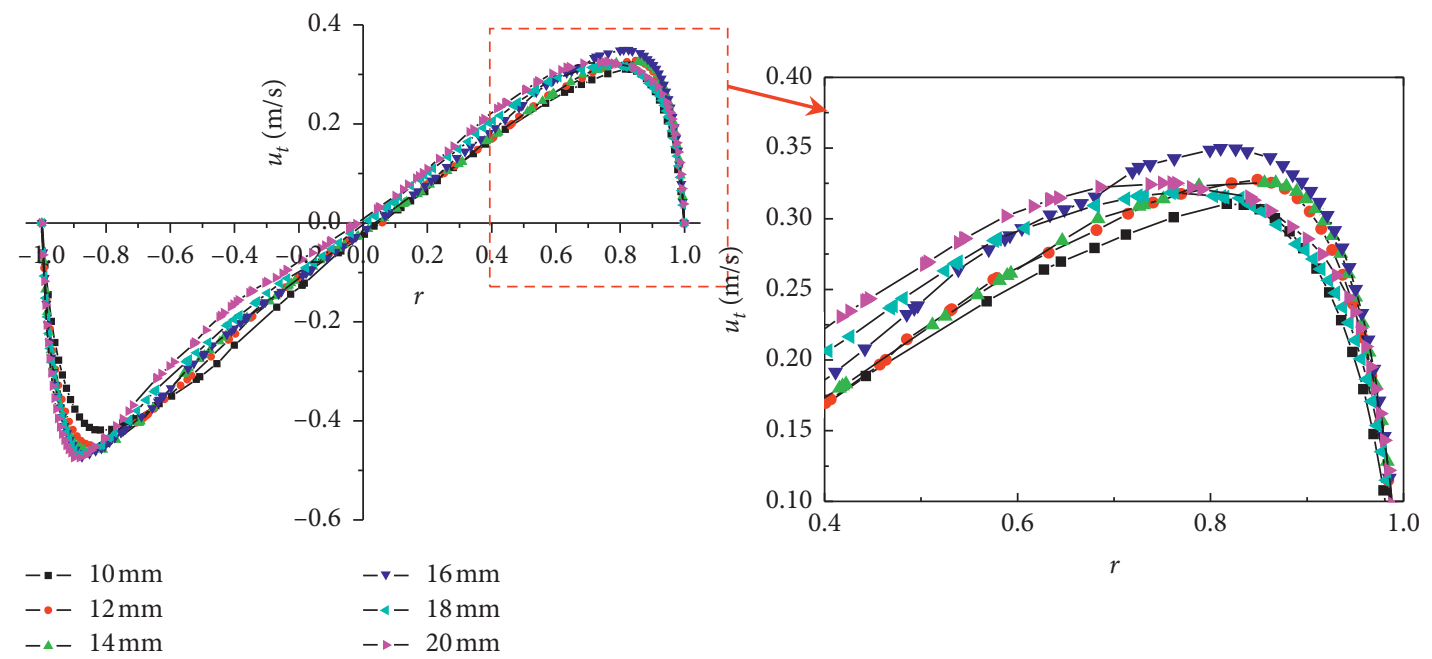

(b)

Figure 11: Tangential velocity distribution of $\mathrm{Y}_{3}$ section at different cylinder diameters. (a) Absolute radial quantity. (b) Radial dimensionless.

TABle 2: Maximum tangential velocity at different cylinder diameters.

\begin{tabular}{lcccccc}
\hline Cylinder diameters $(\mathrm{mm})$ & 10 & 12 & 14 & 16 & 18 & 20 \\
Maximum tangential velocity $(\mathrm{m} / \mathrm{s})$ & 0.3105 & 0.3255 & 0.3253 & 0.3499 & 0.3185 & 0.3252 \\
\hline
\end{tabular}

separation performance; under the research conditions in this section, the length of the separator cylinder is $90 \mathrm{~mm}$, and the aspect ratio is now between 5 and 5.6.

\subsection{The Influence of Cylinder Length on Separator} Performance. Through the previous analysis, it is found that there is the best aspect ratio to make the separation performance of the separator the best. In order to further verify this conclusion, this section focuses on the influence of the length of the cylinder on the performance of the separator, and seven cylinder lengths are selected for research, $60 \mathrm{~mm}$, $70 \mathrm{~mm}, 80 \mathrm{~mm}, 90 \mathrm{~mm}, 100 \mathrm{~mm}, 110 \mathrm{~mm}$, and $120 \mathrm{~mm}$, respectively. When changing the length of the cylinder, keep the distance between the inlet, the air outlet, and the top of the cylinder body unchanged, and keep the distance between the oil outlet and the bottom of the cylinder unchanged.

Figure 14 shows the structure and distribution of flow field in the separator in different cylinder lengths. As you can see from the picture, different cylinder length causes differences in the structure of the flow field inside the separator. When $l_{2}=60 \mathrm{~mm}$, there are a top circulation flow (A), a short-circuit flow of the gas outlet (B), and a bottom circulation flow $(\mathrm{C})$, and the bottom circulation flow is a single vortex structure. Through the previous analysis, it can be concluded that the separation performance is not strong at this time. With the increase of the length of the cylinder, the bottom circulating flow area is stretched (see the comparison 


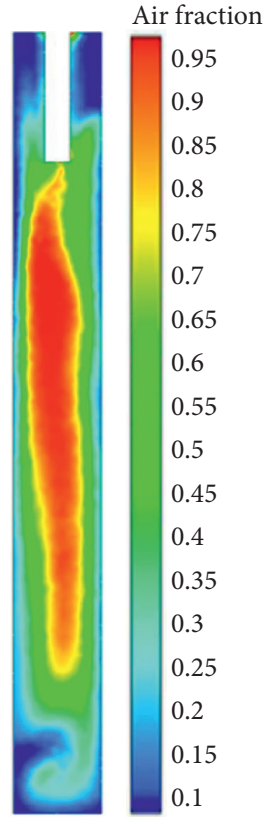

(a)

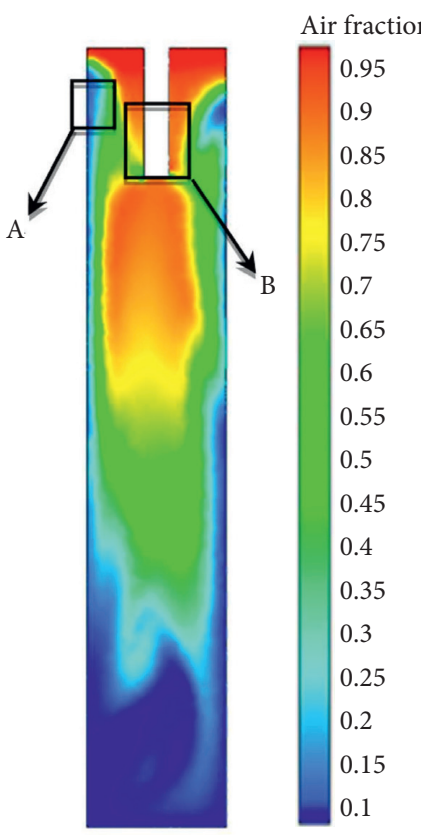

(d)

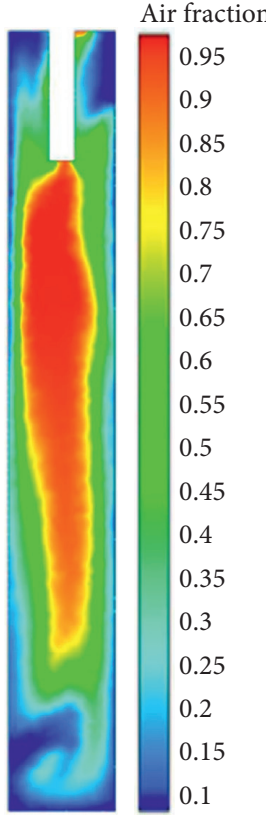

(b)

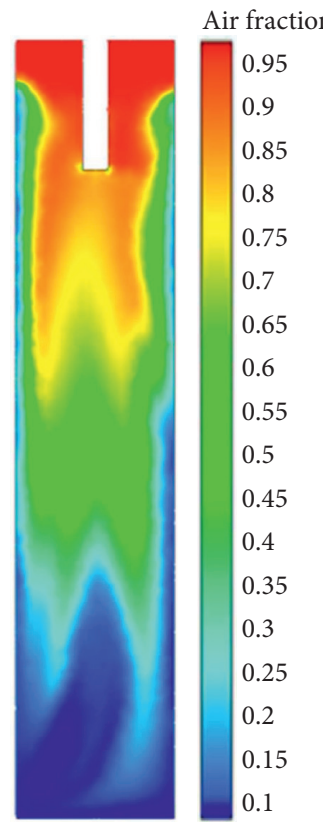

(e)

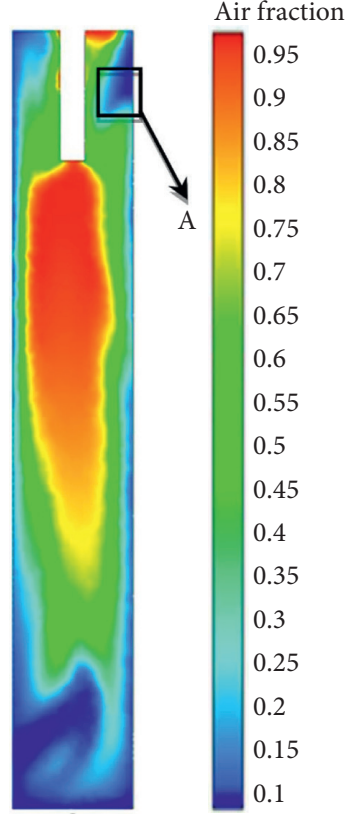

(c)

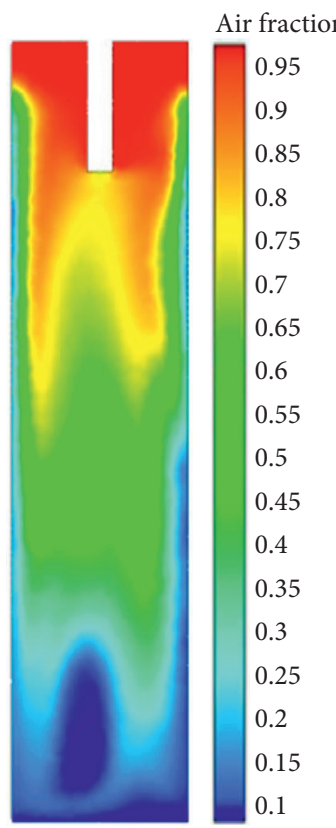

(f)

Figure 12: Gas volume distribution of each section at different cylinder diameters. (a) $d_{\text {sep }}=10 \mathrm{~mm}$. (b) $d_{\text {sep }}=12 \mathrm{~mm}$. (c) $d_{\text {sep }}=14 \mathrm{~mm}$. (d) $d_{\text {sep }}=16 \mathrm{~mm}$. (e) $d_{\text {sep }}=18 \mathrm{~mm}$. (f) $d_{\text {sep }}=20 \mathrm{~mm}$.

of C, D, and E) and the single vortex structure is split into a double vortex structure (see F and G). Meanwhile, the separation effect is enhanced. Unlike the effect of cylinder diameter, with the increase of cylinder length, the double vortex structure at the bottom of the cylinder does not merge but gradually separates and finally forms the distribution form of $\mathrm{H}$ and I. The circulation flow near the bottom of the cylinder is gradually compressed and draws closer to the bottom of the cylinder (see the comparison of G, K, L, and I). In this case, the separation effect should be further evaluated in combination with the gas volume distribution. In addition, the vortex in the central region of the cylinder gradually expands along the axial direction, and its impact range expands, as shown in M. In conclusion, the flow field structure of $l_{2} \geq 90 \mathrm{~mm}$ is superior to that of $l_{2} \leq 80 \mathrm{~mm}$.

Figure 15 shows the cross-sectional velocity distribution of separator $\mathrm{Y}_{3}$ under different cylinder lengths. From Figure 15(a), it can be seen that the overall axial velocity distribution shape is consistent with a "W" shape. When $l_{2} \leq 80 \mathrm{~mm}$, the axial velocity in the central area is flat and the 


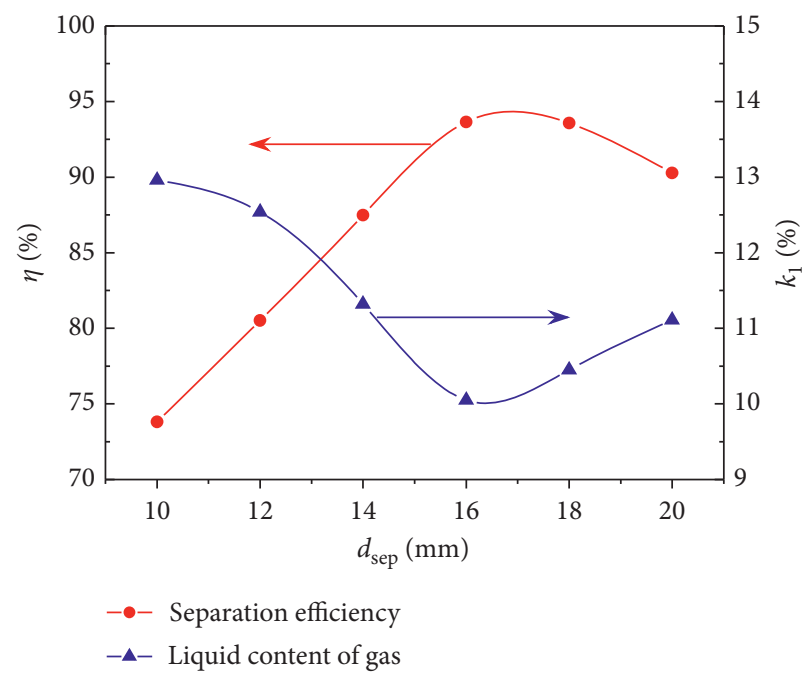

FIgURE 13: Effect of cylinder diameters on separation efficiency and gas-liquid content.

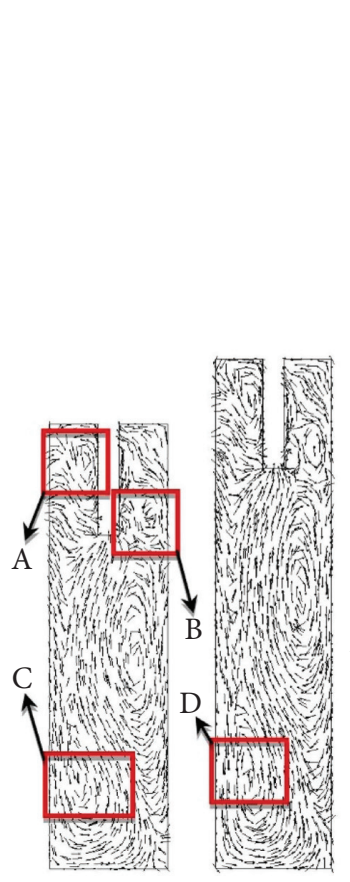

(a) (b)

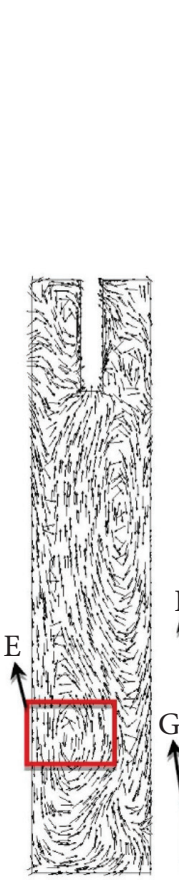

(c)

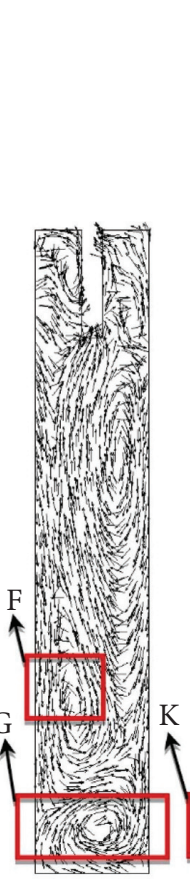

(d)

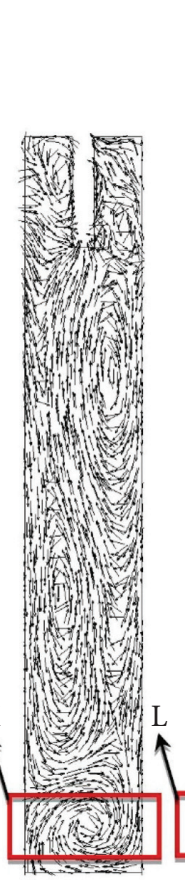

(e)

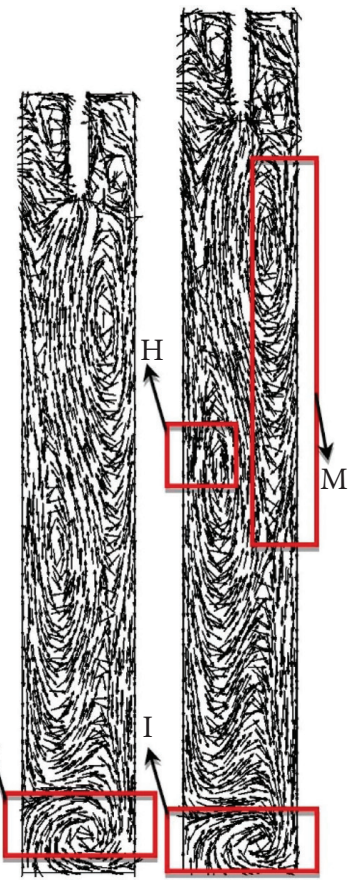

(f)

(g)

Figure 14: Separator flow field at different cylinder lengths. (a) $l_{2}=60 \mathrm{~mm}$. (b) $l_{2}=70 \mathrm{~mm}$. (c) $l_{2}=80 \mathrm{~mm}$. (d) $l_{2}=90 \mathrm{~mm}$. (e) $l_{2}=100 \mathrm{~mm}$. (f) $l_{2}=110 \mathrm{~mm}$. (g) $l_{2}=120 \mathrm{~mm}$.

radial width is larger. As the length of the cylinder increases, the radial width in the central area becomes smaller, and the maximum axial velocity also becomes smaller. At the same time, by analyzing the axial velocity of the right side of the wall, it can be seen that the separator with a small cylinder has a greater downward axial velocity and the residence time of the gas in the separator becomes shorter. At this time, the separation performance is not good at this point. When $l_{2} \geq 100 \mathrm{~mm}$, the axial speed is close and the separation performance at this time needs further study. Figure 15(b) shows the tangential velocity distribution of the $\mathrm{Y}_{3}$ section of the separator in different cylinder lengths. The tangential velocity of various cylinder lengths is distributed in an inverted " $\mathrm{S}$ " shape, with opposite directions on both sides of the center. Due to the influence of the boundary layer of the wall, it first increases and then decreases in the radial direction. Judging from the absolute value of the tangential velocity, when $l_{2}=60 \mathrm{~mm}$, the $\mathrm{Y}_{3}$ cross-section tangential velocity is the largest, and the separation effect is stronger. As the cylinder length increases, the tangential velocity 


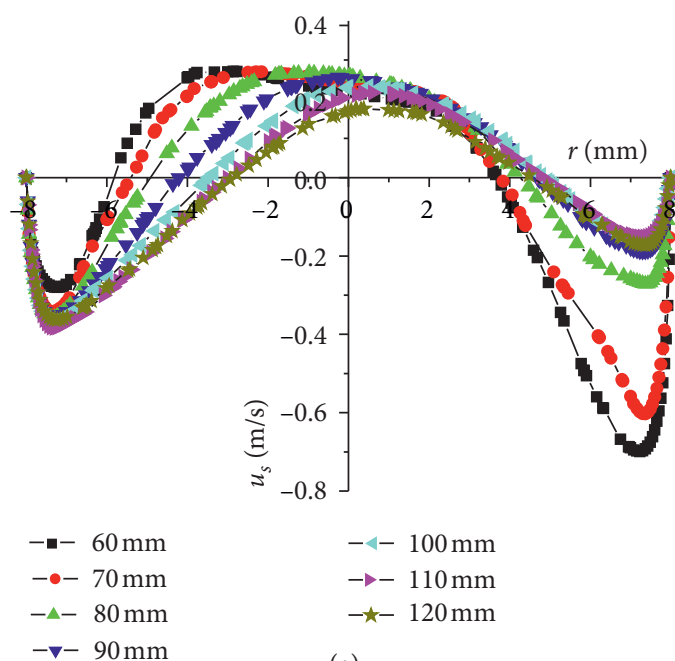

(a)

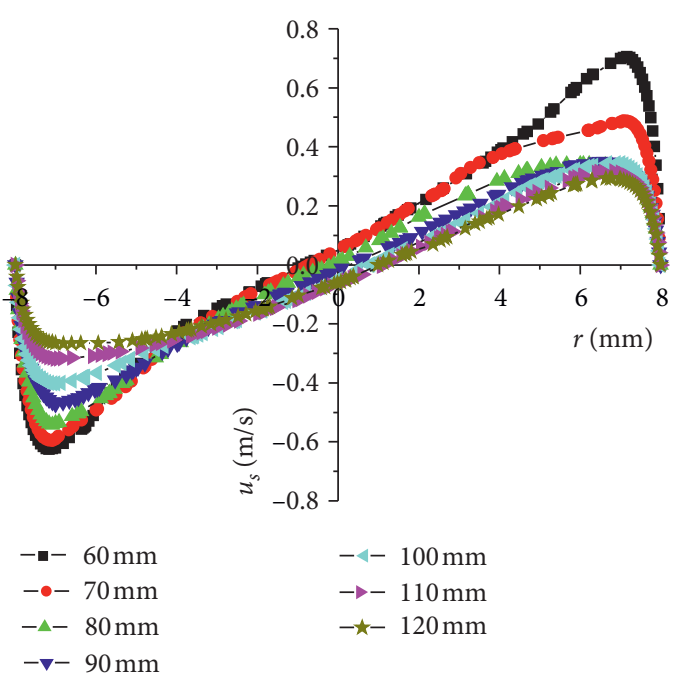

(b)

FIgUre 15: Axial velocity distribution of $Y_{3}$ sections at different cylinder lengths. (a) Axial speed. (b) Tangential speed.

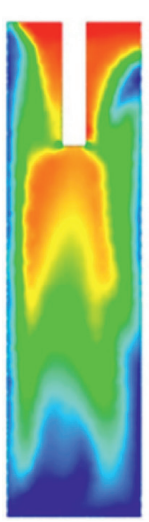

(a)

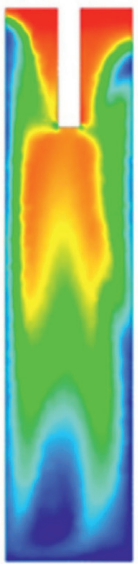

(b)

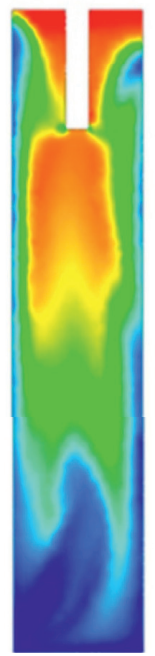

(c)

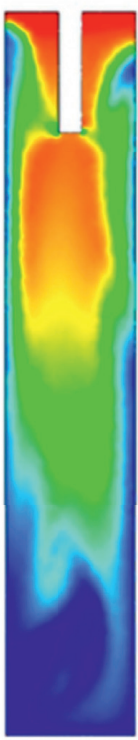

(d)

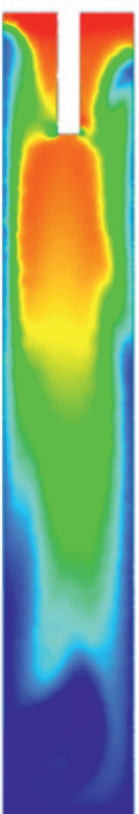

(e)

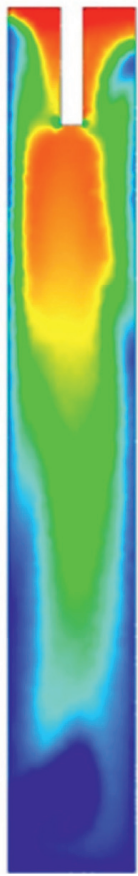

(f)
Air fraction
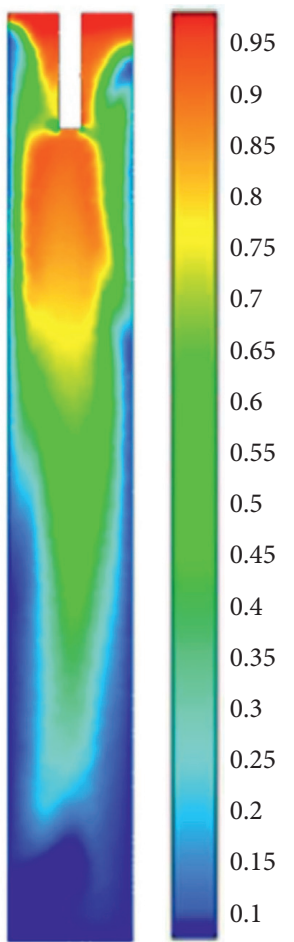

(g)

FIGURE 16: Gas volume distribution nephogram at different cylinder lengths. (a) $l_{2}=60 \mathrm{~mm}$. (b) $l_{2}=70 \mathrm{~mm}$. (c) $l_{2}=80 \mathrm{~mm}$. (d) $l_{2}=90 \mathrm{~mm}$. (e) $l_{2}=100 \mathrm{~mm}$. (f) $l_{2}=110 \mathrm{~mm}$. (g) $l_{2}=120 \mathrm{~mm}$.

decreases and the separation effect weakens, but this does not indicate that the separation effect of the separator is the best when $l_{2}=60 \mathrm{~mm}$. Meanwhile, due to the shortness of the cylinder body, the residence time of the gas is short, and the separated gas will flow to the oil outlet with the main flow, thereby reducing the separation performance.
Figure 16 is a cloud diagram of the gas volume distribution in the separator under different cylinder lengths. It can be seen from the figure that the length of the cylinder has little effect on the gas volume distribution structure in the separator. A large amount of gas accumulates in the central area to form an obvious gas core column with the highest gas 


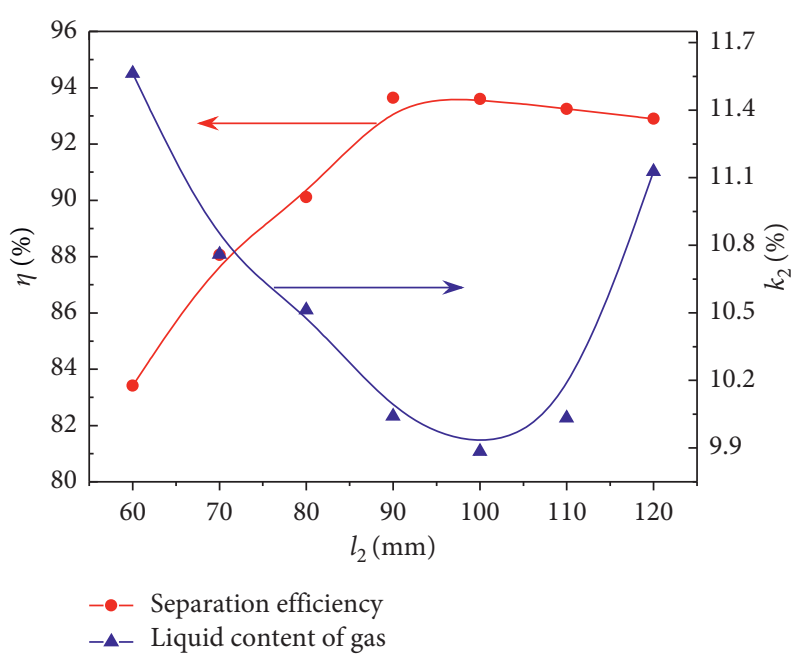

Figure 17: Effect of cylinder lengths on separation efficiency and gas-liquid content.

TABLE 3: Influence trend of structure parameters on separation efficiency.

\begin{tabular}{lccccccc}
\hline Parameter & \multicolumn{11}{c}{ Value } \\
\hline Length of cylinder $l_{2} /(\mathrm{mm})$ & 60 & 70 & 80 & 90 & 100 & 110 & 120 \\
$l_{2} / d_{\text {sep }}$ & 3.8 & 4.4 & 5.0 & 5.6 & 6.3 & 6.9 & 7.5 \\
$\begin{array}{l}\text { Separation efficiency change } \\
\text { trend }\end{array}$ & $\nearrow$ & $\nearrow$ & $\nearrow$ & - & $\searrow$ & $\searrow$ & $\searrow$ \\
$\begin{array}{l}\text { Cylinder diameter } d_{\text {sep }} /(\mathrm{mm}) \\
l_{2} / d_{\text {sep }}\end{array}$ & 10 & 12 & 14 & 16 & 18 & 20 & \\
$\begin{array}{l}\text { Separation efficiency change } \\
\text { trend }\end{array}$ & 9.0 & 7.5 & 6.4 & 5.6 & 5.0 & 4.5 & \\
\hline
\end{tabular}

concentration at the top; when the minimum length of the cylinder is $l_{2}=60 \mathrm{~mm}$, the internal flow is not stable enough. The circulation flow at the bottom of the cylinder is a single vortex structure and is close to the center position, resulting in an " $\mathrm{M}$ "-shaped distribution of the gas core column and bringing the gas in the central area to the vicinity of the oil outlet pipe, resulting in higher gas concentration near the oil outlet pipe, and the separation performance decreases. As the length of the cylinder increases, when $l_{2}=90 \mathrm{~mm}$, the circulation flow at the bottom of the cylinder changes from a single vortex structure to a double vortex structure, thereby enhancing the separation performance, the gas concentration at the bottom of the separator decreases, and the bottom circulation flow suffers compression, and the area of the air core column expands and gradually extends to the bottom of the cylinder, thereby reducing the separation performance. In addition, the gas distribution structure near the top outlet pipe remains unchanged.

Figure 17 shows the separation efficiency of the separator and distribution of the separator under different cylinder lengths. As the length of the cylinder increases, the separation efficiency first increases, reaches the maximum when $l_{2}=90 \mathrm{~mm}$, and then decreases but remains relatively stable; when the cylinder length is between $90 \mathrm{~mm}$ and $110 \mathrm{~mm}$, gas-liquid-holding rate of the gas outlet maintains a low level. If the separator cylinder is too long or too short, the separation performance will decrease. Under the research conditions in this section, the diameter of the separator cylinder is $16 \mathrm{~mm}$. When the separation efficiency is at its maximum and the gas-liquid-holding rate is small $\left(l_{2}=90 \mathrm{~mm}\right)$, the aspect ratio is 5.6 . Combining the above analysis, it can be considered that the separator shows the best performance when the ratio of length to diameter is between 5 and 6 .

3.4. The Influence of Cylinder Length-to-Diameter Ratio on the Performance of Separator. Combining the influence of the analyzed cylinder diameter and cylinder length on the flow field and performance of the separator, Table 3 shows the optimal value of the structural parameters for the separation efficiency of the separator. Among them, " $\nearrow$ " means that the changing trend from this parameter to the right parameter is increasing, " $\searrow$ " means that the trend is decreasing, and “-”" is the extreme point; the optimal value point of each structural parameter can be clearly seen from the table; that is, within the scope of this paper, there is an optimal aspect ratio; that is, when the separator's aspect ratio is between 5 and 6 , the separator has the best separation performance.

\section{Conclusion}

(1) As the diameter of the cylinder increases, the separation efficiency increases, and when $d_{\text {sep }}=16 \mathrm{~mm}$ and $d_{\text {sep }}=18 \mathrm{~mm}$, the separation efficiency of the separator is the largest, about $93 \%$; there is liquid entrainment inside the separator under different cylinder diameters, and the liquid holdup rate of the gas is the lowest when the separation efficiency is maximum.

(2) As the length of the cylinder increases, the separation efficiency first increases, reaches the maximum when $l_{2}=90 \mathrm{~mm}$, and then decreases. When the cylinder length is between $90 \mathrm{~mm}$ and $110 \mathrm{~mm}$, liquid holdup rate of the gas at the gas outlet maintains a low level. If the separator cylinder is too long or too short, the separation performance will decrease.

(3) There is an optimal length-to-diameter ratio, that is, the separation performance of the separator is the best when the length-to-diameter ratio of the separator is between 5 and 6 .

\section{Data Availability}

All the data generated or analyzed during this study are included in this article.

\section{Conflicts of Interest}

The authors declare that they have no financial and personal relationship with other people or organizations that can inappropriately influence their work. 


\section{References}

[1] J. S. Lin, C. J. Chang, and J. G. Yang, Aero Engine Design Manual, Navigate Industrical Press, Beijing, China, 2002.

[2] L. Gomez, R. Mohan, and O. Shoham, "Swirling gas-liquid two-phase flow-experiment and modeling part I: swirling flow field," Journal of Fluids Engineering, vol. 126, no. 6, pp. 935-942, 2004.

[3] R. Hreiz, C. Gentric, N. Midoux, R. Lainé, and D. Fünfschilling, "Hydrodynamics and velocity measurements in gas-liquid swirling flows in cylindrical cyclones," Chemical Engineering Research and Design, vol. 92, no. 11, pp. 2231-2246, 2014.

[4] R. Hreiz, R. Lainé, J. Wu, C. Lemaitre, C. Gentric, and D. Fünfschilling, "On the effect of the nozzle design on the performances of gas-liquid cylindrical cyclone separators," International Journal of Multiphase Flow, vol. 58, pp. 15-26, 2014.

[5] T.-C. Hsiao, S.-H. Huang, C.-W. Hsu, C.-C. Chen, and P.-K. Chang, "Effects of the geometric configuration on cyclone performance," Journal of Aerosol Science, vol. 86, pp. 1-12, 2015.

[6] Y. Fan, J. Wang, Z. Bai, J. Wang, and H. Wang, "Experimental investigation of various inlet section angles in mini-hydrocyclones using particle imaging velocimetry," Separation and Purification Technology, vol. 149, pp. 156-164, 2015.

[7] M. A. Reyes-Gutiérrez, L. R. Rojas-Solórzano, J. C. MarínMoreno, A. J. Meléndez-Ramírez, and J. Colmenares, "Eulerian-eulerian modeling of disperse two-phase flow in a gas-liquid cylindrical cyclone," Journal of Fluids Engineering, vol. 128, no. 4, pp. 832-837, 2006.

[8] M.-F. Kang, N. C. Hoyt, J. Kadambi, and Y. Kamotani, "Study of gas core behavior of passive cyclonic two-phase separator for microgravity applications," Microgravity Science and Technology, vol. 26, no. 3, pp. 147-157, 2014.

[9] M. Saidi, R. Maddahian, B. Farhanieh, and H. Afshin, "Modeling of flow field and separation efficiency of a deoiling hydrocyclone using large eddy simulation," International Journal of Mineral Processing, vol. 112-113, no. 112-113, pp. 84-93, 2012.

[10] M. Saidi, R. Maddahian, and B. Farhanieh, "Numerical investigation of cone angle effect on the flow field and separation efficiency of deoiling hydrocyclones," Heat and Mass Transfer, vol. 49, no. 2, pp. 247-260, 2013.

[11] J. J. Meng, Y. Luo, G. Yan, and J. M. Feng, "Numerical simulation on the effect of inlet-collision in oil-gas separator used for air-conditioning system," Applied Mechanics and Materials, vol. 448-453, pp. 3378-3381, 2013.

[12] R. Guizani, H. Mhiri, and P. Bournot, "CFD study of the effect of rotation speed on dynamic air separator flow characteristics and pressure drop," in Proceedings of the 2014 5th International Renewable Energy Congress. IREC 2014, Hammamet, Tunisia, March 2014.

[13] C. M. Yang, Mechanism Model Research and Numerical Simulation on Dynamic Pressure Type Gas-Oil Separator, Harbin Engineering University, Harbin, China, 2013.

[14] Y. G. Lu and J. P. Hu, "Numerical simulation for air/oil separator of aero-engine," Applied Mechanics and Materials, vol. 510, pp. 197-201, 2014.

[15] R. Guizani, I. Mokni, H. Mhiri, and P. Bournot, "CFD modeling and analysis of the fish-hook effect on the rotor separator's efficiency," Powder Technology, vol. 264, pp. 149-157, 2014.

[16] S. L. Van, "Influence of inlet angle on flow pattern and performance of gas-liquid cylindrical cyclone separator," Particulate Science and Technology, vol. 35, pp. 555-564, 2017.
[17] S. Wang, H. Li, R. Wang, X. Wang, R. Tian, and Q. Sun, "Effect of the inlet angle on the performance of a cyclone separator using CFD-DEM," Advanced Powder Technology, vol. 30, no. 2, pp. 227-239, 2019.

[18] W. B. Zhu, L. Hu, and X. B. Zhang, "“The effects of the lower outlet on the flow field of small gas-liquid cylindrical cyclone," Proceedings of the Institution of Mechanical Engineers, Part C: Journal of Mechanical Engineering Science, vol. 233, no. 4, pp. 1989-1996, 2018.

[19] A. Ghasemi, M. Shams, and M. M. Heyhat, "A numerical scheme for optimizing gas liquid cylindrical cyclone separator," Proceedings of the Institution of Mechanical Engineers, Part E: Journal of Process Mechanical Engineering, vol. 231, no. 4, pp. 1-13, 2016.

[20] D. Li, A. Buffo, W. Podgórska, D. L. Marchisio, and Z. Gao, "Investigation of droplet breakup in liquid-liquid dispersions by CFD-PBM simulations: the influence of the surfactant type," Chinese Journal of Chemical Engineering, vol. 25, no. 10, pp. 1369-1380, 2017.

[21] M. Siadaty, S. Kheradmand, and F. Ghadiri, "Study of inlet temperature effect on single and double inlets cyclone performance," Advanced Powder Technology, vol. 28, no. 6, pp. 1459-1473, 2017.

[22] L. Huang, S. Deng, Z. Chen, J. Guan, and M. Chen, "Numerical analysis of a novel gas-liquid pre-separation cyclone," Separation and Purification Technology, vol. 194, pp. 470-479, 2018.

[23] Y. Li, G. Qin, Z. Xiong, Y. Ji, and L. Fan, “The effect of particle humidity on separation efficiency for an axial cyclone separator," Advanced Powder Technology, vol. 30, no. 4, pp. 724-731, 2019.

[24] T. Yue, J. Chen, J. Song et al., "Experimental and numerical study of upper swirling liquid film (USLF) among gas-liquid cylindrical cyclones (GLCC)," Chemical Engineering Journal, vol. 358, pp. 806-820, 2019.

[25] L. Yang, J. Zhang, Y. Ma, J. Xu, and J. Wang, "Experimental and numerical study of separation characteristics in gas-liquid cylindrical cyclone," Chemical Engineering Science, vol. 214, p. 115362, 2020.

[26] X. B. Zhang, L. Lang, X. F. Zhang, H. Q. Lv, and N. Gao, "Performance prediction model of dynamic pressure oil-air separator," International Journal of Aerospace Engineering, vol. 2021, p. 6665869, 2021.

[27] F. M. Erdal, Local Measurements and Computational Fluid Dynamics Simulations in a Gas-Liquid Cylindrical Cyclone Separator, The University of Tulsa, Tulsa, Oklahoma, 2001.

[28] R. F. Tian and P. A. Liu, Numerical Heat Transfer and Fluid Flow, Harbin Engineering University Press, Harbin, China, 2015.

[29] M. J. Prince and H. W. Blanch, "Bubble coalescence and break-up in air-sparged bubble columns," AIChE Journal, vol. 36, no. 10, pp. 1485-1499, 1990.

[30] H. Luo and H. F. Svendsen, "Theoretical model for drop and bubble breakup in turbulent dispersions," AIChE Journal, vol. 42 , no. 5, pp. 1225-1233, 1996.

[31] X. B. Zhang, The Research on Performance of Dynamic Pressure Type Gas-Oil Separator in Aero-Engine, Harbin Engineering University, Harbin, China, 2018. 